\title{
Traduire
}

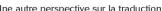

Revue française de la traduction

$240 \mid 2019$

Quand la politique s'en mêle

\section{La « Pérégrination vers l'Ouest » de Xi Jinping}

Ou quand le président chinois tente de se muer en politicien francophone

Kevin Henry

\section{OpenEdition}

\section{Journals}

Édition électronique

URL : http://journals.openedition.org/traduire/1647

DOI : 10.4000/traduire.1647

ISSN : 2272-9992

Éditeur

Société française des traducteurs

Édition imprimée

Date de publication : 20 juin 2019

Pagination : 24-35

ISSN : 0395-773X

\section{Référence électronique}

Kevin Henry, "La «Pérégrination vers l'Ouest » de Xi Jinping », Traduire [En ligne], 240 | 2019, mis en ligne le 20 juin 2019, consulté le 05 février 2020. URL : http://journals.openedition.org/traduire/1647 DOI : 10.4000/traduire.1647 


\section{La "Pérégrination vers I'Ouest" de Xi Jinping}

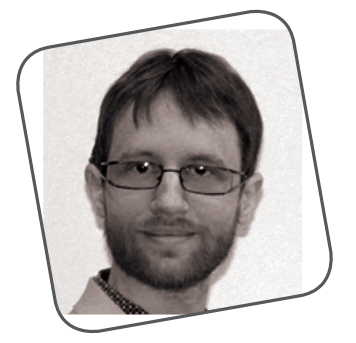

\section{Ou quand le président chinois tente de se muer en politicien francophone}

\section{Kevin Henry}

Depuis son avènement en 1949, le régime communiste chinois recourt amplement à la propagande pour galvaniser les masses et répandre la bonne parole. Toutefois, I'ascension en 2012-2013 du président $X i$ Jinping a vu une inflation galopante de ces entreprises de matraquage idéologique.

Dans la continuité de cet élan propagandiste, un autre phénomène à l'œuvre en Chine est le prosélytisme inédit pratiqué vers l'étranger. Partenaire essentiel dans le contexte de la mondialisation, le pays, dont les intentions restent opaques, inquiète les autres nations. À grand renfort de soft power, l'Empire du Milieu tente donc, au-delà des arguments économiques, de séduire la planète en mettant en avant la richesse et le succès de sa culture, mais aussi de son modèle politique et de son idéologie.

La publication multilingue de l'ouvrage doctrinal en deux volumes 《习近平谈治国理政》LaGouvernance de la Chine (Xi2014a/b, $2018 \mathrm{a} / \mathrm{b}$ ), qui réunit des retranscriptions de discours, allocutions, communiqués et autres entretiens de Xi Jinping depuis sa nomination, est un exemple symptomatique de cette fièvre prosélyte qui gagne le pays. Les Éditions en langues étrangères (外文出版社), qui patronnent le recueil, sont une branche de l'Administration 
chinoise de publication et de diffusion en langues étrangères (中国外文出版发行事业局), organe dépendant du département de la Publicité du Comité central du Parti communiste chinois (中共中央 宣传部). L'identité des traducteurs qui ont participé à cette entreprise sans précédent reste inconnue, mais il ne fait aucun doute que les versions étrangères ont été effectuées par des équipes locales de langue maternelle chinoise du Bureau de compilation et de traduction du Comité central du PCC (中共中央编译局), ce qui explique en partie leur hétérogénéité stylistique et idiomatique.

Dans le présent article, nous examinerons la rhétorique de La Gouvernance de la Chine dans sa version française avec les outils de l'analyse du discours (Amossy 2006, CharterisBlack 2014, Chilton 2004) et à travers le prisme des translational norms (Toury 2012). À l'heure où les investissements considérables que l'Empire du Milieu consacre à ses forces armées suscitent l'interrogation de l'Occident, il semble bienvenu de déconstruire les discours du président chinois consacrés à la politique militaire; nous chercherons ainsi à montrer comment la traduction a tenté de surmonter la difficulté d'allier surenchère nationaliste destinée au public chinois et volonté d'apaisement sur la scène internationale. Les quatre documents, réunis au sein du chapitre XII du volume II consacré à l'"Édification des forces armées», sont les suivants:

- 《充分发挥政治工作对强军兴军的生命线作用》 《Mettre pleinement en valeur le rôle du travail politique en tant que ligne vitale dans le renforcement et la montée en gamme de notre armée» (Xi 2018a: 499-505);

- 《全面实施改革强军战略》 《Mettre en œuvre sur tous les plans la stratégie de renforcement militaire par la réforme» (Xi 2018a: 506-514);

- 《加快形成军民融合深度发展格局》 《Accélérer la mise en place d'une architecture de développement en profondeur de l'intégration civilo-militaire» (Xi 2018a: 515-519);

- 《把强军事业不断推向前进》 《Promouvoir constamment l'édification d'une armée puissante» (Xi 2018a: 520-525).

Nous compléterons notre exposé en confrontant la traduction officielle et celle effectuée par nos étudiants de master 1 en traduction chinois-français lors du séminaire "Ateliers de traduction». Suivant une approche socioconstructiviste, cette unité d'enseignement, qui regroupe pendant deux semaines tous les étudiants de master 1 en Traduction et en Interprétation de 
l'université de Mons (toutes finalités et langues confondues), propose une simulation d'un bureau de traduction multilingue professionnel, où les étudiants sont tenus de participer à toutes les étapes du traitement d'un projet dans le domaine de la traduction ou de l'interprétation. Dans l'optique d'une recherche critique, nos étudiants ont reçu la consigne de restituer au mieux la rhétorique de $X_{i}$ Jinping en français, tout en se conformant à sa visée propagandiste. Par cet exercice, qui reproduit au plus près les conditions de travail des traducteurs chinois originaux, nous estimons être en mesure de comparer de manière pertinente et efficace les sensibilités particulières de traducteurs sinophones et francophones dans un projet explicitement prosélyte.

\section{Analyse}

Notre analyse s'articulera autour des trois composantes majeures de la rhétorique aristotélicienne classique:

- l'éthos, qui représente le style que doit adopter l'orateur pour capter l'attention et gagner la confiance de l'auditoire, afin de se rendre crédible et sympathique;

- le logos, qui correspond à la logique, au raisonnement et au mode de construction de l'argumentation et s'adresse à l'esprit rationnel et objectif de l'interlocuteur;

- le pathos, qui rassemble les techniques stylistiques visant à susciter des passions, des désirs et des émotions et s'adresse à la sensibilité de l'auditoire.

\section{1) L'adaptation à I'auditoire et la construction de l'éthos}

L'un des traits qui frappent immédiatement le lecteur de La Gouvernance de la Chine touche à l'architecture argumentative des allocutions de $X i$ Jinping. Les pratiques discursives du chinois, radicalement différentes de celles du français, auraient pu faire craindre une certaine confusion. Et cela à tort, puisque des phrases parfois très longues de l'orateur sont souvent coupées. Ce réordonnancement rhétorique a pour effet d'accroitre la crédibilité de l'auteur auprès du public en rendant son discours plus rationnel et cohérent. On note toutefois que ce rehaussement de l'éthos de Xi Jinping par le biais de cette mise en scène discursive est moins accusé dans la traduction officielle que dans la version proposée par les étudiants, qui se sont montrés 
très soucieux de (re)structurer et d'«alléger»le discours du président, quitte à supprimer parfois des passages jugés redondants. C'est notamment le cas dans l'exemple suivant, extrait du deuxième texte:

中央国家机关、地方各级党委和政府要强化大局观念, 把支持深化国防和 军队改革当作分内的事, 拿出一些特殊措施和倾斜政策, 主动帮助解决好 退役军人、职工安置工作, 党政军民齐心协力, 共同落实深化国防和军队 改革各项任务, 推动全面实施改革强军战略不断取得新的进展, 为实现中 国梦、强军梦作出新的更大的贡献。(Xi 2018b)

Les organismes d'État relevant de l'autorité centrale ainsi que les comités du Parti et les gouvernements locaux aux divers échelons doivent renforcer leur conscience de l'intérêt général, considérer comme le leur le soutien à l'approfondissement de la réforme de la défense nationale et de l'armée, adopter des mesures spéciales et des politiques préférentielles afin d'aider de leur propre initiative la réinsertion des militaires et employés démobilisés. Le Parti, le gouvernement ainsi que les autorités militaires et civiles doivent conjuguer leurs efforts pour mettre à exécution les diverses tâches destinées à l'approfondissement de la réforme de la défense nationale et de l'armée, pour obtenir sans cesse de nouveaux progrès dans l'application de la stratégie de renforcement militaire par la réforme, et pouvoir contribuer toujours davantage à la réalisation du rêve chinois et à celle du rêve du renforcement militaire. (Xi 2018a: 514)

Il est du devoir des organes centraux de l'État, des comités locaux du Parti et du gouvernement de renforcer le concept d'intérêt public, de soutenir le renforcement de la réforme de la défense nationale et de l'armée. Il faut adopter des mesures particulières et mener des politiques préférentielles pour aider activement à résoudre le problème de retraite des vétérans et de la réaffectation du personnel. Le Parti, le gouvernement, le corps militaire et le peuple devront travailler ensemble pour renforcer et promouvoir la pleine mise en œuvre de la stratégie de réforme et renforcement de l'armée, faire des progrès tactiques pour atteindre de nouveaux objectifs et apporter de nouvelles contributions plus importantes à la réalisation du rêve chinois et militaire. (Ateliers de traduction 2019')

1. Conformément au règlement général sur la protection des données (RGPD), les traductions réalisées par nos étudiants dans la cadre des "Ateliers de traduction» resteront anonymes. II est également à noter qu'elles sont livrées ici dans leur état brut, sans correction des éventuelles erreurs de langue. 
La variété lexicale et syntaxique est aussi l'une des techniques auxquelles les équipes de traducteurs ont recouru le plus souvent pour relever le discours. En effet, alors que Xi Jinping, en bon orateur chinois, ne s'émeut pas de ressasser les mêmes termes, le français abhorre généralement la répétition. Par conséquent, là où les propos de Xi Jinping abondent en réitérations, les traducteurs ont soigneusement veillé à ne pas les reproduire, les étudiants belges se montrant plus sensibles à la diversification des solutions. Par exemple, dans le quatrième texte (Xi 2018a: 520-525), le terme fourre-tout 发展 «développer/ développement», dont on relève vingt occurrences, est traduit par «évolution» (1 item), «développer/développement» $(4+13)$ ou $\varnothing$ (2) dans la version officielle, mais par «évolution» (2), «promouvoir» (2), «avancement» (3), «développement» (2), «croissance» (1), «mise au point» (1), «nourrir» (1) ou $\varnothing$ (8) dans la version de nos étudiants. Cet exemple illustre une autre stratégie facilement applicable, la suppression du terme inopportunément répété. On remarquera toutefois que cette règle ne s'applique pas aux anaphores, très fréquentes chez $X_{i}$ Jinping. Ainsi, dans le troisième discours (Xi 2018a: 515-519), le syntagme 推进军民融合深度发展, qui introduit cinq paragraphes sur huit, est-il rendu systématiquement "afin de promouvoir le développement en profondeur de l'intégration civilo-militaire»par les traducteurs chinois et "pour une intégration civilo-militaire approfondie» par nos étudiants francophones; pour accentuer la progression argumentative, ces derniers y ajoutent cependant des connecteurs logiques («également», «de plus», «enfin»).

Dans tous les cas, les manipulations effectuées servent à "localiser», alléger et polir le discours de l'Autre en le rendant plus policé, cartésien et ordonné. Xi Jinping apparaît ainsi comme un tribun «à la française» prototypique et fantasmé: avisé et maître de sa communication, pondéré et mesuré.

\section{2) La modulation du pathos et les figures de style}

Pour réussir à convaincre son public de la pertinence de son argumentation, l'orateur recourt, entre autres, au pathos: il cherche à susciter une certaine émotion auprès de son auditoire afin de le rapprocher de ses opinions. 
Dans notre cas, la difficulté à laquelle sont confrontés les traducteurs tient à l'hétérogénéité des publics cibles originaux et à leur non-adéquation avec ceux de la version française. En effet, le livre La Gouvernance de la Chine, destiné à persuader un lectorat non sinophone des bienfaits de la pensée de Xi Jinping, est intégralement constitué d'allocutions et de propos prononcés dans des circonstances variées. Dès lors, vaut-il mieux choisir la fidélité au cadre spatio-temporel initial, au risque d'aliéner le lecteur final par l'évocation de sentiments qui lui sont étrangers, ou l'adaptation à un nouvel allocutaire composite, en s'exposant alors à la dénaturation du ton original? Ce questionnement, qui rappelle l'opposition dressée par Lawrence Venuti (1995) entre domestication et foreignization, rejoint la séparation qu'opère Christiane Nord (1997/2008: 64-70) entre la «traduction documentaire», qui, bien que destinée aux lecteurs de la culture cible, témoigne d'une interaction communicative dans la culture source, et la "traduction instrumentale», qui vise une interaction en culture cible fondée sur une interaction en culture source. Concernant les quatre textes analysés ici, nous qualifierons leurs traductions d' "hétéro-fonctionnelles», selon la typologie de Nord: leur intégration dans La Gouvernance de la Chine leur assigne des fonctions (référentielle, expressive, appellative et phatique) similaires, mais non identiques à celles des textes sources.

Tout l'enjeu d'une analyse critique des discours sur l'«Édification des forces armées " sera ainsi de déterminer sous quelles modalités les accents martiaux, potentiellement menaçants, de Xi Jinping ont été transmis en français. Nous avons déjà constaté comment la réorganisation de la structure argumentative, la diversification du vocabulaire et la suppression de redites contribuent à rehausser l'éthos du président chinois, en y apportant une touche de rationalité. Ce même appareil stylistique sert tout autant à feutrer le ton péremptoire de Xi Jinping qu'à rendre sa rhétorique belliqueuse et ronflante moins intimidante pour le public francophone. Tant les traducteurs chinois que nos étudiants francophones natifs nous ont semblé avoir relevé cette gageure, quoiqu'avec une sophistication et une finesse différentes. Un exemple patent de cette tendance au polissage stylistique peut être trouvé dans le passage suivant, tiré du quatrième texte: 
[...] 中国人民珍爱和平, 我们决不搞侵略扩张, 但我们有战胜一切侵略的 信心。我们绝不允许任何人、任何组织、任何政党、在任何时候、以任何 形式、把任何一块中国领土从中国分裂出去，谁都不要指望我们会吞下损 害我国主权、安全、发展利益的苦果。人民军队要坚决维护中国共产党领 导和我国社会主义制度, 坚决维护国家主权、安全、发展利益, 坚决维护 地区和世界和平。( Xi 2018b)

Le peuple chinois chérit la paix. Nous ne nous livrerons pas à l'agression ou à l'expansion, mais nous avons la confiance de pouvoir vaincre tous les agresseurs. Nous ne permettrons à quiconque, à aucune organisation, ni à aucun parti politique, de séparer une quelconque partie du territoire chinois, à quelque moment que ce soit et sous quelque forme que ce soit. Personne ne peut s'attendre à ce que nous acceptions sans broncher un préjudice à la souveraineté, à la sécurité et aux intérêts en matière de développement de la Chine. L'armée populaire doit fermement sauvegarder la direction du Parti communiste chinois et le régime socialiste de notre pays, résolument défendre la souveraineté nationale et les intérêts en matière de développement du pays, ainsi que la paix régionale et mondiale. (Xi 2018a: 522-523)

Le peuple chinois est épris de paix. Nous ne viserons jamais l'invasion ni l'expansion, mais nous avons bon espoir de pouvoir repousser tout ennemi. Nous ne laisserons aucun peuple, organisation, ou parti politique, à aucun moment, et en aucun cas, s'emparer d'une partie de notre territoire. Nous ne vacillerons pas face aux menaces qui nuisent à notre souveraineté, à notre sécurité et à notre développement. L'Armée populaire doit renforcer la direction du Parti communiste et du système socialiste chinois, protéger la souveraineté, la sécurité et les intérêts de notre croissance, et garantir résolument la paix régionale et mondiale. (Ateliers de traduction 2019)

Pour obtenir l'adhésion d'un public, s'appuyer sur la doxa, c'est-à-dire sur le bon sens commun et sur les opinions présumées de l'auditoire, s'avère primordial. Pour ce faire, l'orateur habile n'hésitera pas à user de figures de style et de formules toutes faites: les premières, par leur caractère potentiellement original, attirent l'attention; les secondes, lieux privilégiés du stéréotype, acquièrent une légitimité de leur répétition. Le discours politique est connu pour tirer parti de ces deux stratégies, surtout de la deuxième, fondement de la «langue de bois». Xi Jinping confirme cette tendance: depuis son investiture, il multiplie les effets d'annonce en psalmodiant à qui mieux mieux les mêmes slogans. II n'est pas non plus avare de citations, comme 
l'atteste le premier discours, d'où sont notamment tirés les items suivants; leur origine littéraire est explicitée, dans toutes les versions, dans des notes en bas de page:

“秉纲而目自张，执本而末自从。”

“为将之道, 当先治心。”

“令行禁止, 王者之师。”

“明制度于前, 重威刑于后。”

“为威不强还自亡，立法不明还自伤” (Xi 2018b)

"Les mailles du filet s'ouvrent quand on en tire la corde principale, et les membres obéissent eux-mêmes au corps dominant.»

"Le principe à suivre pour un général, c'est d'abord cultiver son esprit.»

"Une puissante armée exécute strictement les ordres émis.»

"Publier préalablement le règlement avant de punir sérieusement le coupable.»

"Un prestige en déclin mènera à l'autodestruction et une législation confuse causera préjudice à soi-même.» (Xi 2018a: 499-505)

"Tout comme le filet ne tient qu'à une seule corde, c'est tout l'équipage qui suit le timonier.»

"Qui se veut général cultive son esprit.»

"C'est en obéissant aux ordres sans broncher que sera garantie la victoire de l'armée.»

"Pour punir sévèrement les coupables, publiez d'abord un règlement.»

"Une autorité en déclin mènera à sa propre destruction, et une législation confuse se portera préjudice.» (Ateliers de traduction 2019)

Le lecteur observera que, suivant notre conseil, les étudiants ont tenté de conserver le rythme des formulations originales, en s'efforçant de couler leur traduction dans le moule de l'alexandrin.

Souvent les expressions figées auxquelles fait appel le président prennent la forme de chengyu, un type de phrasème propre au chinois dont l'une des caractéristiques principales est son rythme quadrisyllabique; la suite de plusieurs de 
ces séquences produit alors un effet de scansion particulier (Henry 2016). Invariablement, on constate que la traduction ne tient pas compte de leur valeur gnomique et prosodique. Ce lissage doxique et cette réduction stylistique du pouvoir proverbial des chengyu procèdent donc d'une démarche d'accommodation. Dans la citation suivante, issue du premier texte, le propos qui s'articule autour du terme 党 «Parti» se voit renforcé par l'inclusion de quatre chengyu successifs:

二是要把党性原则在全军牢固立起来。坚持党性原则是共产党人的根本政 治品格, 是政治工作的根本要求。政治工作必须坚持党的原则第一、党的 事业第一、人民利益第一, 在党言党、在党忧党、在党为党, 把爱党、忧 党、兴党、护党落实到工作各个环节。(Xi 2018b)

Deuxièmement, les principes régissant l'esprit du Parti doivent être solidement implantés dans toute l'armée. S'en tenir aux principes régissant l'esprit du Parti est la qualité politique fondamentale des membres du Parti et l'exigence fondamentale du travail politique. Dans le travail politique, il faut assurer de manière absolue la primauté aux principes du Parti, à la cause du Parti et aux intérêts du peuple. Les membres du Parti doivent garder à Pesprit leur identité, se préoccuper du Parti, le servir, concrétiser dans toutes leurs activités leur affection et leur préoccupation envers le Parti, et œuvrer pour sa prospérité et sa protection. (Xi 2018a: 501)

Deuxièmement, il faut fermement imposer le principe de conscience de Parti à l'ensemble de l'armée. Insister sur ce principe est l'essence même du communisme, et constitue l'exigence fondamentale du travail politique. L'activité politique doit d'abord insister sur les principes du Parti, en servir la cause, et faire passer les intérêts du peuple avant tout: le Parti par le Parti pour le Parti. (Ateliers de traduction 2019)

On notera que, à contre-pied de la version officielle un peu verbeuse, nos étudiants belges - certes en condensant excessivement le propos - ont essayé de restituer la valeur d'insistance du rythme psalmodique en insérant en fin de paragraphe une formule-choc.

\section{3) Les imperfections}

Si la traduction des textes de La Gouvernance de la Chine recourt à une acclimatation, passant par une mise en relief du logos, une mitigation du pathos et une atténuation de la doxa 
chinoise originale, en vue de conférer à $X i$ Jinping un éthos de confiance et de sérieux, il faut reconnaitre que l'opération présente plusieurs faiblesses qui pourraient compromettre tout l'effort prosélyte déployé.

Mentionnons d'abord la disparité des solutions proposées pour des formulations ou des concepts pourtant identiques. Ce problème peut sans doute s'expliquer par un manque de coordination au sein de l'équipe de traducteurs, ou par un travail de relecture insuffisant. Ce trait s'observe aussi, quoique peut-être dans une moindre mesure, chez nos étudiants.

Un point plus grave concerne les erreurs. Elles résultent parfois de la tentative maladroite de traduire un terme vague et mal défini. L'une des occurrences les plus troublantes concerne le terme 建设 (39 items). Le sens de ce verbe employé en architecture pour «bâtir» ou "ériger» s'avère fuyant lorsqu'il est appliqué à un groupe humain, par exemple une armée: il oscille entre "former» et "entraîner» (y compris politiquement) d'un côté, "renforcer» et "consolider» de l'autre. Or le texte français parle très majoritairement d' "édification» et de "construction», termes équivoques qui sonnent un peu faux ${ }^{2}$. Nos étudiants, quant à eux, ont utilisé une plus grande variété lexicale: outre les solutions des traducteurs chinois, très minoritaires, figurent ainsi les termes «avancement», "mise en œuvre», "mise en place», «consolidation», "élaboration», "croissance», "progression», «instauration» et "renforcement», avec une légère préférence pour ce dernier terme ou pour la suppression pure et simple. La plupart des autres cas introduisent des connotations divergentes par l'emploi de tournures faisant sourciller le lecteur, voire d'imperfections qui confinent parfois au ridicule. Les chengyu ne sont pas épargnés, comme le montre l'exemple suivant, issu du quatrième texte:

[…] 全党全军全国各族人民要大力弘扬军爱民、民拥军的光荣传统, 不断 发展坚如磐石的军政军民关系。(Xi 2018b)

Tout le Parti, toute l'armée et tout notre peuple multiethnique doivent faire pleinement valoir les glorieuses traditions de l'amour de l'armée pour le peuple et du soutien du peuple à l'armée, et ne cesser

2. L'emploi du verbe 建设 dans ce contexte pourrait de fait se rapprocher de l'emploi similaire en français de «construire» dans le sens de «former», «entraîner» ou «éduquer» dans le jargon marxiste. 
de développer les relations monolithiques entre l'armée, le gouvernement et le peuple. (Xi 2018a:525)

Que ce soit le Parti, l'armée ou la nation dans son ensemble, toutes ethnies confondues, chacun d'entre nous doit perpétuer la glorieuse tradition d'une armée prenant soin du peuple et des masses soutenant leurs soldats, et sans cesse nourrir l'union, solide comme un roc, entre gouvernement et armée, et entre armée et peuple. (Ateliers de traduction 2019)

Si l'on peut se douter que bon nombre de ces incorrections proviennent du fait que la traduction officielle est l'œuvre de locuteurs non natifs évoluant en quasi-autarcie au service d'une agence étatique d'un pays lointain, il reste qu'elles peuvent être préjudiciables à la promotion de cette publication à l'étranger. On soulignera du reste que les étudiants francophones ne sont pas à l'abri, tant s'en faut, de ce genre d'erreurs, qui s'expliquent dans leur cas par une mécompréhension du discours original (certes très complexe et rébarbatif).

\section{Conclusion ouverte}

Tout en réitérant l'importance que revêt La Gouvernance de la Chine dans le nouveau positionnement idéologique imposé par le maître actuel de Pékin, nous soulignerons en conclusion le professionnalisme accru dont font preuve les organismes officiels dans leur cause prosélyte. En effet, pour toute personne qui ne connaît pas le chinois, le recueil se présente rédigé dans une langue relativement correcte et soignée. En outre, en comparant la version française publiée avec les productions de nos étudiants, en dépit des quelques trouvailles ingénieuses qu'ont pu faire ces derniers, nous ne sommes pas certains, eu égard à la difficulté (l'obscurité?) des discours de Xi Jinping, qu'une équipe de traducteurs ou de relecteurs francophones natifs s'en serait mieux sortie.

Malgré les tentatives tangibles d'émousser le plus possible les aspérités d'un style redondant, grandiloquent et empreint de sous-entendus, on peut toutefois légitimement se demander si le lecteur francophone pourra se départir de sa méfiance envers un recueil ne cherchant plus à cacher son caractère propagandiste. 
Docteur en Langues, Lettres et Traductologie, Kevin Henry est depuis septembre 2017 maître de conférences en Langue et Culture chinoises à la faculté de Traduction et d'Interprétation de l'université de Mons (Belgique). Traducteur spécialisé dans les sciences humaines, il a livré une version française des ouvrages chinois Le Modèle Alibaba de Ying Lowrey (Nuvis 2016) et Fei Xiaotong, I'homme qui voulait comprendre la Chine de Li Shengming (Nuvis 2017); il prépare actuellement une traduction d'essais du philosophe Liang Shuming. Ses domaines de recherche touchent la traduction chinois-français et français-chinois, la critique de la traduction, l'analyse du discours et la traduction littéraire.

\section{SOURCES CITÉES}

AMOSSY Ruth, L'Argumentation dans le discours, Paris, Armand Colin, 2006.

CHARTERIS-BLACK Jonathan, Analysing Political Speeches: Rhetoric, Discourse and Metaphor, Houndmills (Royaume-Uni), Palgrave Macmillan, 2014.

CHILTON Paul, Analysing Political Discourse: Theory and Practice, Londres, Routledge, 2004. HENRY Kevin, «Les chengyu du chinois: caractérisation de phrasèmes hors norme», in Yearbook of Phraseology, 1, 2016, p. 99-126.

NORD Christiane, La Traduction: une activité ciblée. Introduction aux approches fonctionnalistes, traduit de l'anglais par Beverly Adab, Arras (France), Artois Presses Université, 1997/2008.

TOURY Gideon, Descriptive Translation Studies and Beyond (Revised Edition). Amsterdam (PaysBas), John Benjamins, 2012.

XI Jinping, La Gouvernance de la Chine, traducteurs anonymes, Pékin, Foreign Language Press, 2014a.

习近平 XI Jinping, 《习近平谈治国理政》, 北京 Beijing, 外文出版社 Foreign Language Press, 2014b, version Kindle, consulté en mars 2019.

XI Jinping, La Gouvernance de la Chine II, traducteurs anonymes, Pékin, Foreign Language Press, 2018a.

习近平 XI Jinping, 《习近平谈治国理政第二卷》, 北京 Beijing, 外文出版社 Foreign Language Press, 2018b, version Kindle, consulté en mars 2019. 